Check for updates

Cite this: RSC Adv., 2018, 8, 17497

Received 8th February 2018

Accepted 30th April 2018

DOI: 10.1039/c8ra01224e

rsc.li/rsc-advances

\section{Effects of template molecules on the structures and luminescence intensities of a series of porous Tb- MOFs based on the 2-nitroterephthalate ligand $\dagger$}

\author{
Yi-Xia Ren, (D) Xiao-Long Zhao, Zhi-Xiang Wang, Yong Pan, Hui-Ping Li, \\ Fei-Yan Wang, Shao-Feng Zhu and Chen-Hui Shao
}

\begin{abstract}
Four novel porous $\mathrm{Tb}(\mathrm{III})$ metal-organic frameworks (Tb-MOFs) have been designed and prepared hydrothermally from 2-nitroterephthalate (2- $\mathrm{H}_{2}$ ntp), namely $\left\{\left[\mathrm{Tb}(2-n t p)_{1.5}\left(\mathrm{H}_{2} \mathrm{O}\right)\right] \cdot \mathrm{H}_{2} \mathrm{O}\right\}_{n} \quad(1), \quad\{[\mathrm{Tb}(2-$ ntp) $\left.)_{2}\left(\mathrm{H}_{2} \mathrm{O}\right)\right] \cdot 4,4^{\prime}-\mathrm{Hbipy}_{n}(2),\left\{\left[\mathrm{Tb}(2-\mathrm{ntp})_{2}\left(\mathrm{H}_{2} \mathrm{O}\right)\right] \cdot 2,4-\mathrm{Hbipy}\right\}_{n}(3)$, and $\left\{\left[\mathrm{Tb}(2-\mathrm{ntp})_{2}\left(\mathrm{H}_{2} \mathrm{O}\right)\right] \cdot\left(1,4-\mathrm{H}_{2} \mathrm{bbi}\right)_{0.5}\right\}_{n}(4)$ $\left(4,4^{\prime}\right.$-bipy $=4,4^{\prime}$-bipyridine; 2,4-bipy $=2,4$-bipyridine; 1,4 -bbi $=1,4$-bisbenzimidazole $)$. X-ray diffraction structural analyses show these Tb-MOFs are porous and are based on $\mathrm{Tb}^{3+}$ ions and 2nitroterephthalate, in which water molecules (1) or protonated $\mathrm{N}$-donor ligands (2-4) exist as templates. The fluorescence properties of complexes 1-4 could be associated with the characteristic peaks of $\mathrm{Tb}$ (III) ions, and the existence of different guest molecules affects the intensities of peaks, which means that these could be potential fluorescence materials, with intensities adjusted using guests.
\end{abstract}

\section{Introduction}

Porous metal-organic frameworks (MOFs) have attracted increasing attention due to their diversified structures and potential applications to heterogeneous catalysis, selective guest adsorption, the immobilization of ions, energy storage and so on. ${ }^{1}$ In the pores of MOFs, there are solvents or other small molecules, such as ions, anions, amino-compounds and so on. ${ }^{2}$ Some of these can be exchanged with other ions or molecules, but some are templates which can't be exchanged and are located in the pores. ${ }^{3}$ Lanthanide ions have high coordination numbers and large ionic radii, resulting in varieties of lanthanide porous MOF materials that exhibit more outstanding properties toward ionic selection and discrimination, nanoparticle catalysis, guest exchange and adsorption. ${ }^{4}$ As a new species, lanthanide-based metal-organic frameworks (Ln-MOFs) attract more research attention in the fields of material, synthetic and inorganic chemistry. ${ }^{5}$ Of the lanthanides, $\mathrm{Eu}^{3+}$ and $\mathrm{Tb}^{3+}$ ions possess good fluorescence properties, so $\mathrm{Eu} / \mathrm{Tb}-\mathrm{MOF}$ have good fluorescence detection abilities and wide applications in fluorescence probes, fluorescence discrimination and sensing: ${ }^{6}$

Multi-carboxylate ligands have been employed in the preparation of MOFs due to the varied coordination modes of the

College of Chemistry and Chemical Engineering, Laboratory of New Energy and New Function Materials, Yan'an University, Yan'an 716000, P. R. China. E-mail: renyixia1@163.com

$\dagger$ Electronic supplementary information (ESI) available: Selected bond distances and angles are listed in Tables S1-4. TG curves and PXRD patterns are available for 1-4. CCDC 1815608-1815611 for complexes 1-4. For ESI and crystallographic data in CIF or other electronic format see DOI: 10.1039/c8ra01224e carboxyl group. ${ }^{7}$ Bicarboxylate functional ligands, such as terephthalate and its derivatives, have been used in the construction of a great deal of MOFs as rigid spacers. ${ }^{8}$ Furthermore, different auxiliary co-ligands containing nitrogen, such as bipyridine, also have been used to adjust structural features and properties. ${ }^{9}$ In the paper, we used a simple terephthalate derivative, namely 2-nitroterephthalate, to design and prepare successfully a series of Tb-MOFs featuring different guest molecules. Interestingly, for these complexes, the N-donor coligands are not coordinated to Tb ions, but are located in the pores as template molecules.

\section{Experimental}

\section{Materials and measurements}

All chemicals were commercially available and used as received without further purification. Elemental analyses (CHN) were performed using a Vario EL elemental analyzer. FT-IR spectra were recorded from $\mathrm{KBr}$ pellets over the range of $4000-400 \mathrm{~cm}^{-1}$ on a Nicolet Avatar 360 FT-IR spectrometer. Thermogravimetric curves were measured using a Netzsch STA449 F3 analyzer at a heating rate of $10^{\circ} \mathrm{C} \mathrm{min}^{-1}$ from room temperature to $900{ }^{\circ} \mathrm{C}$ in air. Fluorescence measurements were carried out with a SHIMADZU RT5301PC spectrofluorophotometer. The lifetimes of the excited states were measured with an Edinburgh Instruments FLS920 fluorescence spectrophotometer. X-ray powder diffraction (XRPD) intensities were measured at $293 \mathrm{~K}$ using a D8 Advance (Bruker) diffractometer $(\mathrm{Cu}-\mathrm{K} \alpha ; \lambda=1.54056$ $\AA$ ). The simulated PXRD patterns were calculated from the single crystal diffraction data using PowderCell. 


\section{Syntheses}

Synthesis of $\left\{\left[\mathrm{Tb}(2-n t p)_{1.5}\left(\mathrm{H}_{2} \mathrm{O}\right)\right] \cdot \mathrm{H}_{2} \mathrm{O}\right\}_{n}$ (1). A mixture of $\mathrm{Tb}(\mathrm{OAc})_{3} \cdot 6 \mathrm{H}_{2} \mathrm{O}(0.021 \mathrm{~g}, 0.075 \mathrm{mmol})$, and 2- $\mathrm{H}_{2}$ ntp $(0.020 \mathrm{~g}, 0.1$ mmol) was added to $3 \mathrm{~mL}$ of $\mathrm{H}_{2} \mathrm{O}$. The mixture was stirred for 10 minutes and was placed in a $23 \mathrm{~mL}$ Teflon-lined stainless steel autoclave and heated at $140{ }^{\circ} \mathrm{C}$ for $72 \mathrm{~h}$. The autoclave was cooled over a period of $18 \mathrm{~h}$ in air. The colorless stick-like crystals of 1 were collected via filtration, washed with ethanol, and dried in air (yield: 65\%, based on $\mathrm{Tb}$ ). Elemental analyses: calcd for $\mathrm{C}_{48} \mathrm{H}_{32} \mathrm{~N}_{6} \mathrm{O}_{43} \mathrm{~Tb}_{4}$ (1): C: $28.59 ; \mathrm{H}: 1.60 ; \mathrm{N}: 4.17 \%$; found C: 28.92; H: 1.21; N: 4.39\%. IR ( $\left.\mathrm{cm}^{-1}\right): 3454(\mathrm{~m}) ; 2914(\mathrm{w}) ; 2357$ (w); 1620 (s); 1577 (vs.); 1545 (s); 1521 (s); 1494 (vs.); 1491 (s); 1375 (s); $929(\mathrm{~m}) ; 858(\mathrm{~m}) ; 783(\mathrm{~m})$.

Synthesis of $\left\{\left[\mathbf{T b}(2-n t p)_{2}\left(\mathbf{H}_{2} \mathbf{O}\right)\right] \cdot 4,4^{\prime}-\mathbf{H b i p y}\right\}_{n}$ (2). A mixture of $\mathrm{Tb}(\mathrm{OAc})_{3} \cdot 6 \mathrm{H}_{2} \mathrm{O}(0.021 \mathrm{~g}, 0.075 \mathrm{mmol}), 2-\mathrm{H}_{2} \mathrm{ntp}(0.020 \mathrm{~g}, 0.1$ $\mathrm{mmol}$ ) and 4,4'-bipy ( $0.016 \mathrm{~g}, 0.1 \mathrm{mmol})$ was added to $3 \mathrm{~mL}$ of $\mathrm{H}_{2} \mathrm{O}$. The mixture was stirred for 10 minutes and was placed in a $23 \mathrm{~mL}$ Teflon-lined stainless steel autoclave and heated at $140{ }^{\circ} \mathrm{C}$ for $72 \mathrm{~h}$. The autoclave was cooled over a period of $18 \mathrm{~h}$ in air. The colorless stick-like crystals of 2 were collected via filtration, washed with ethanol, and dried in air (yield: $58 \%$, based on $\mathrm{Tb}$ ). Elemental analyses: calcd for $\mathrm{C}_{26} \mathrm{H}_{17} \mathrm{~N}_{4} \mathrm{O}_{13} \mathrm{~Tb}(2)$ : C: 41.51; H: 2.28; N: 7.45\%; found C: 41.87; H: 1.99; N: 7.64\%. IR $\left(\mathrm{cm}^{-1}\right): 3450(\mathrm{~m}) ; 2912(\mathrm{w}) ; 2360(\mathrm{w}) ; 1574(\mathrm{~s}) ; 1524(\mathrm{~s}) ; 1408(\mathrm{~s}) ;$ 1341 (s); 977 (m); 850 (m); 808 (m); $750(\mathrm{w})$.

Synthesis of $\left\{\left[\mathrm{Tb}(2-\mathrm{ntp})_{2}\left(\mathrm{H}_{2} \mathrm{O}\right)\right] \cdot 2,4-\mathrm{Hbipy}\right\}_{n} \quad$ (3). The synthesis process for complex 3 is similar to that for 2 , except for the use of 2,4-bipy in place of 4,4'-bipy when adding the materials. The colorless stick-like crystals of 3 were collected via filtration, washed with ethanol, and dried in air (yield: 43\%, based on $\mathrm{Tb}$ ). Elemental analyses: calcd for $\mathrm{C}_{26} \mathrm{H}_{17} \mathrm{~N}_{4} \mathrm{O}_{13} \mathrm{~Tb}(3)$ : C: $41.51 ; \mathrm{H}: 2.28 ; \mathrm{N}: 7.45 \%$; found C: $41.32 ; \mathrm{H}: 2.05 ; \mathrm{N}: 7.88 \%$. IR (cm $\left.{ }^{-1}\right): 3404(\mathrm{~m}) ; 2916(\mathrm{w}) ; 2372(\mathrm{w}) ; 1608$ (m); 1578 (vs.); 1516 (m); 1398 (vs.); 1342 (m); 982 (w); 829 (w); 769 (w).

Synthesis of $\left\{\left[\mathbf{T b}(2-n t p)_{2}\left(\mathbf{H}_{2} \mathrm{O}\right)\right] \cdot\left(1,4-\mathrm{H}_{2} \mathbf{b b i}\right)_{0.5}\right\}_{n}$ (4). The synthesis process for complex 4 is similar to that for 2 , except that 1,4 -bbi is used in place of $4,4^{\prime}$-bipy when adding the materials. The faint yellow stick-like crystals of $\mathbf{4}$ were collected via filtration, washed with ethanol, and dried in air (yield: $20 \%$, based on Tb). Elemental analyses: calcd for $\mathrm{C}_{23} \mathrm{H}_{15} \mathrm{~N}_{4} \mathrm{O}_{13} \mathrm{~Tb}(4)$ : C: 38.67; H: 2.12; N: 7.84\%; found C: 38.79; H: 2.37; N: 7.45\%. IR $\left(\mathrm{cm}^{-1}\right)$ : $3449(\mathrm{~m}) ; 2918(\mathrm{w}) ; 2360(\mathrm{w}) ; 1580(\mathrm{~s}) ; 1522(\mathrm{~s}) ; 1393(\mathrm{~m})$; 1344 (m); $990(\mathrm{w}) ; 831(\mathrm{w}) ; 766(\mathrm{w})$.

\section{$\mathrm{X}$-ray data collection and structural determination}

Single crystal X-ray diffraction analyses of the coordination polymers 1-4 were carried out on a Bruker SMART APEX CCD diffractometer equipped with graphite monochromated Mo-K $\alpha$ radiation $(\lambda=0.71073 \AA)$. Raw data were integrated using the SAINT program. ${ }^{10}$ The structures were solved via direct methods with SHELXS-97 and refined using full-matrix least-squares on $F^{2}$ using SHELXS-97. ${ }^{11}$ An empirical absorption correction was applied using the program SADABS. ${ }^{12}$ All non-hydrogen atoms were refined anisotropically. The hydrogen atoms were set in calculated positions and refined using a riding mode. Parts of the 2-nitro group on 2-ntp ${ }^{2-}$ ligands are positionally disordered in complexes 1-4. Crystallographic details of 1-4 are provided in Table 1, while selected bond distances and angles are listed in Tables S1-4. $\dagger$

\section{Results and discussion}

\section{Crystal structure of 1}

$\mathrm{X}$-ray single-crystal diffraction analysis shows that $\mathbf{1}$ has a threedimensional porous framework with lattice water molecules

Table 1 Crystal data and structural refinement for complexes 1-4

\begin{tabular}{|c|c|c|c|c|}
\hline Complex & 1 & 2 & 3 & 4 \\
\hline Empirical formula & $\mathrm{C}_{48} \mathrm{H}_{32} \mathrm{~N}_{6} \mathrm{O}_{43} \mathrm{~Tb}_{4}$ & $\mathrm{C}_{26} \mathrm{H}_{17} \mathrm{~N}_{4} \mathrm{O}_{13} \mathrm{~Tb}$ & $\mathrm{C}_{26} \mathrm{H}_{17} \mathrm{~N}_{4} \mathrm{O}_{13} \mathrm{~Tb}$ & $\mathrm{C}_{23} \mathrm{H}_{15} \mathrm{~N}_{4} \mathrm{O}_{13} \mathrm{~Tb}$ \\
\hline$M_{\mathrm{W}}$ & 2016.48 & 752.36 & 752.36 & 714.31 \\
\hline$T / \mathrm{K}$ & $296(2)$ & $296(2)$ & $296(2)$ & $296(2)$ \\
\hline Crystal system & Orthorhombic & Monoclinic & Monoclinic & Monoclinic \\
\hline Space group & $C 2 / c$ & $P 2_{1} / n$ & $P 2_{1} / n$ & $P 2_{1} / a$ \\
\hline$a / \AA$ & $16.7852(19)$ & $11.9800(5)$ & $11.7024(8)$ & $15.308(3)$ \\
\hline$b / \AA$ & $11.3430(13)$ & $14.5780(6)$ & $14.5744(9)$ & $15.900(3)$ \\
\hline$c / \AA$ & $15.3991(18)$ & $15.2371(6)$ & $15.8996(11)$ & $11.580(2)$ \\
\hline$\alpha /^{\circ}$ & 90 & 90 & 90 & 90 \\
\hline$\beta /^{\circ}$ & 90 & $105.5310(10)$ & $105.519(11)$ & $118.12(3)$ \\
\hline$\gamma /{ }^{\circ}$ & 90 & 90 & 90 & 90 \\
\hline$V / \AA^{3}$ & $2931.9(6)$ & 2563.91(18) & $2612.9(3)$ & $2485.8(8)$ \\
\hline$Z$ & 2 & 4 & 4 & 4 \\
\hline$D_{\text {calcd }} / \mathrm{g} \mathrm{cm}^{-1}$ & 2.284 & 1.949 & 1.913 & 1.903 \\
\hline$\mu / \mathrm{mm}^{-1}$ & 4.890 & 2.841 & 2.788 & 2.923 \\
\hline$\theta$ range $/^{\circ}$ & 3.42 to 27.64 & 3.11 to 25.01 & 3.00 to 25.01 & 3.16 to 25.01 \\
\hline Reflections collected & 22200 & 25667 & 34170 & 32271 \\
\hline Unique reflections & 3119 & 3716 & 4199 & 3227 \\
\hline GOF & 1.055 & 1.077 & 1.031 & 1.014 \\
\hline$R_{1}[I>2 \sigma(I)]$ & 0.0273 & 0.0266 & 0.0205 & 0.0382 \\
\hline $\mathrm{w} R_{2}$ (all data) & 0.0736 & 0.0605 & 0.0565 & 0.1622 \\
\hline
\end{tabular}




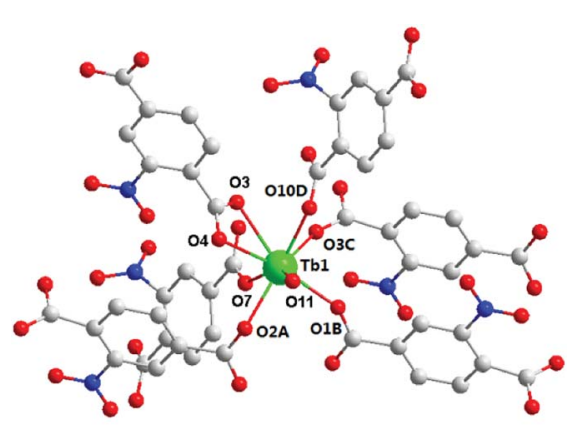

(a)

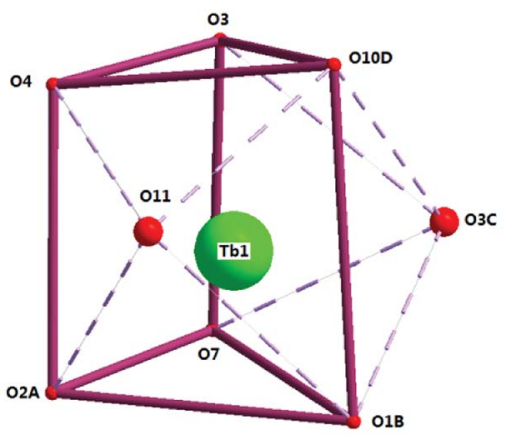

(b)

Fig. 1 The Tb1 coordination environment (a) and polyhedron (b) for complex 1. All H atoms are omitted for clarity. Symmetry codes: (A) 0.5 $-x, 0.5+y, 0.5-z$; (B) $0.5+x, 1.5-y,-0.5+z$; (C) $1-x, y, 0.5-z$; and (D) $x,-1+y, z$.

filling in the pores. The center metal $\mathrm{Tb}^{3+}$ ion is surrounded by eight oxygen atoms from one chelated carboxyl group (O3 and $\mathrm{O} 4)$, five bridged carboxyl groups (O1B, O2A, O3C, O7, and O10D) from six 2-ntp ligands, and one water molecule (O11) (Fig. 1a). The $\mathrm{Tb}^{3+}$ ion polyhedron is in a distorted two-capped triangular prism configuration, in which $\mathrm{O} 3, \mathrm{O} 4$, and $\mathrm{O} 10 \mathrm{D}$, and $\mathrm{O} 7, \mathrm{O} 2 \mathrm{~A}$, and $\mathrm{O} 1 \mathrm{~B}$ construct two surfaces, and $\mathrm{O} 3 \mathrm{C}$ and $\mathrm{O} 11$ are vertex atoms (Fig. $1 \mathrm{~b}$ ). The $\mathrm{Tb}-\mathrm{O}$ bond lengths range from 2.292(2) to 2.601(3) $\AA$, which are in accordance with those found in the other $\mathrm{Tb}$ (III) complexes. ${ }^{13}$

The 2-ntp ${ }^{2-}$ ligand in 1 exhibits two $\mu_{4}$ coordination modes, $\left(\kappa^{2}-\kappa^{1}\right)-\left(\kappa^{1}-\kappa^{1}\right)-\mu_{4}$ and $\left(\kappa^{1}-\kappa^{1}\right)-\left(\kappa^{1}-\kappa^{1}\right)-\mu_{4}($ Chart $1(a)$ and (b)), linking four $\mathrm{Tb}^{3+}$ ions. The adjacent $\mathrm{Tb}^{3+}$ ions (the $\mathrm{Tb} \cdots \mathrm{Tb}$
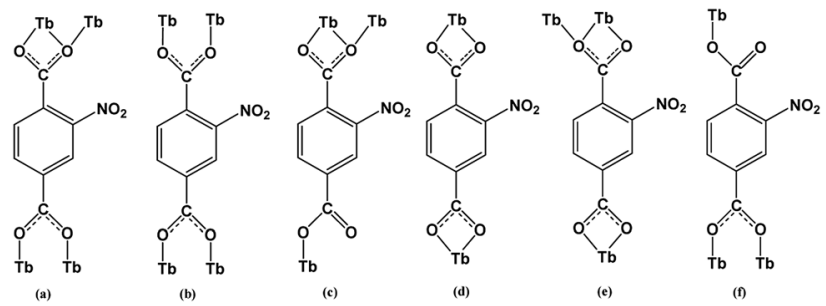

Chart 1 The coordination modes of the $2-n \mathrm{tp}^{2-}$ ligand in complexes 1-4. (a) $\left(\kappa^{2}-\kappa^{1}\right)-\left(\kappa^{1}-\kappa^{1}\right)-\mu_{4}$; (b) $\left(\kappa^{1}-\kappa^{1}\right)-\left(\kappa^{1}-\kappa^{1}\right)-\mu_{4}$; (c) $\left(\kappa^{2}-\kappa^{1}\right)-\left(\kappa^{1}\right)-\mu_{3} ;$ (d) $\left(\kappa^{2}\right)-\left(\kappa^{2}\right)-\mu_{2} ;(e)\left(\kappa^{2}-\kappa^{1}\right)-\left(\kappa^{2}\right)-\mu_{3} ;$ and $(f)\left(\kappa^{1}-\kappa^{1}\right)-\left(\kappa^{1}\right)-\mu_{3}$.



Fig. 2 A one-dimensional chain in 1.

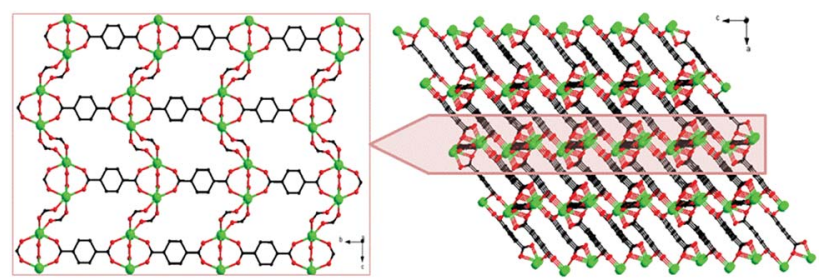

Fig. 3 The two-dimensional network (left) and three-dimensional porous structure (right) of 1 (the nitro groups in the 2-ntp ligands and hydrogen atoms have been omitted for clarity).

distances are 3.96 and $5.23 \AA$ ) are bridged via carboxyl groups, in $\left(\kappa^{2}-\kappa^{1}\right)-\mu_{2}$ and $\left(\kappa^{1}-\kappa^{1}\right)-\mu_{2}$ modes, from 2-ntp ligands to form a one-dimensional chain with two kinds of rings, four-member rings and six-member rings, joining alternately, as shown in Fig. 2. Then the 1D chains extend into a 2D network bridged by 2-ntp ${ }^{2-}$ ligands in the $b c$ plane. Furthermore, the 3D porous structure has been formed through 2 -ntp ${ }^{2-}$ ligands in different directions, in which the guest water molecules (O12) exist

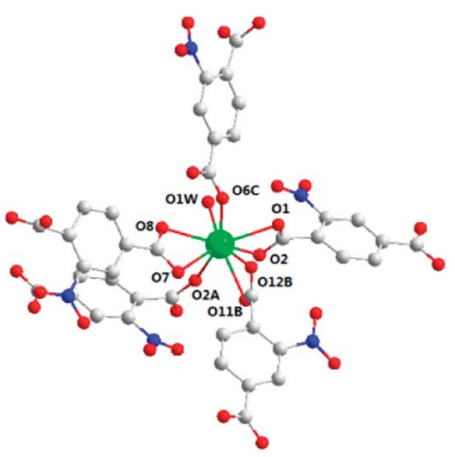

(a)

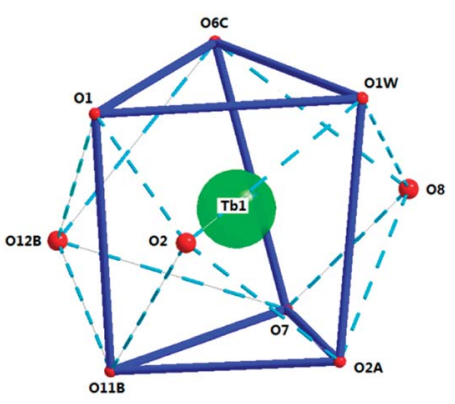

(b)

Fig. 4 The Tb1 coordination environment (a) and polyhedron (b) for complex 2. All H atoms are omitted for clarity. Symmetry codes: (A) $2-x, 1-y,-z$; (B) $1.5-x, 0.5+y, 0.5-z$; and (C) $1.5-x,-0.5+y$, $-0.5-z$. 


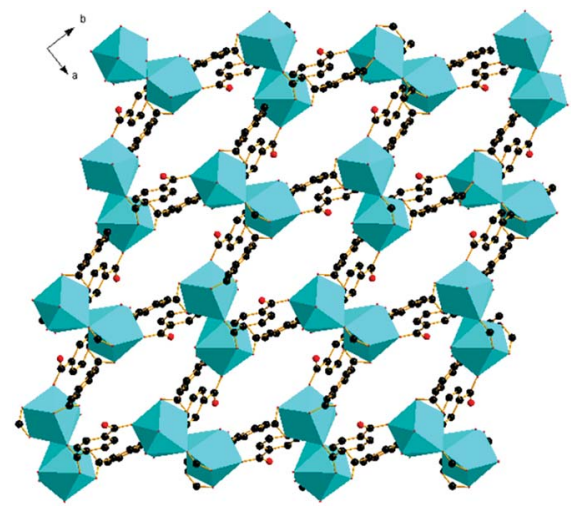

Fig. 5 The two-dimensional structural of 2 from the $c$ axis. The nitro groups and $\mathrm{H}$ atoms are omitted for clarity.

through H-bonds with coordination water molecules (O11) (O11-H11wB $\cdots \mathrm{O} 12: 2.647 \AA$, 164.52 $2^{\circ}$ ) and oxygen atoms (O4 and O6) from the carboxyl groups of ligands (O12-H12wA $\cdots \mathrm{O} 4$ :

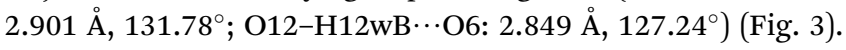

\section{Crystal structures of 2 and 3}

Structural analyses reveal that complexes 2 and 3 crystallize in the same monoclinic system, the $P 2_{1} / n$ space group, and the frameworks of complexes $\mathbf{2}$ and $\mathbf{3}$ are isomorphic, despite having different $\mathrm{N}$-donor molecules as guests in their pores. Here, only complex 2 will be described in detail. In 2 , the Tb1 ion lies in a nine-coordinated three-capped triangular prism configuration, in which $\mathrm{O} 2, \mathrm{O} 8, \mathrm{O} 12 \mathrm{~B}$ are the capping atoms and $\mathrm{O} 1, \mathrm{O} 6 \mathrm{C}$ and $\mathrm{O} 1 \mathrm{~W}$, and $\mathrm{O} 11 \mathrm{~B}, \mathrm{O} 7$, and $\mathrm{O} 2 \mathrm{~A}$ form the two faces of the prism (Fig. 4b). Of the nine coordinated oxygen atoms, six atoms $(\mathrm{O} 1, \mathrm{O} 2, \mathrm{O} 7, \mathrm{O} 8, \mathrm{O} 11 \mathrm{~B}$, and $\mathrm{O} 12 \mathrm{~B})$ are from chelated carboxyl groups of 2-ntp ${ }^{2-}$ ligands, O2A and O6C belong to bridging carboxyl groups, and $\mathrm{O} 1 \mathrm{~W}$ comes from one water molecule (Fig. 4a). The Tb-O bond lengths range from $2.304(2)$ to $2.638(2) \AA$, and the $\mathrm{O}-\mathrm{Tb}-\mathrm{O}$ bond angles are in the range of $50.92(7)$ to $155.74(9)^{\circ}$.

The dimer unit consists of two terbium ions with a $\mathrm{Tb} \cdots \mathrm{Tb}$ distance of $4.23 \AA$, and extends into a three-dimensional porous framework, bridging via 2-ntp ${ }^{2-}$ ligands (Fig. 5). The 2-ntp ${ }^{2-}$ ligands in 2 adopt two coordination modes, $\left(\kappa^{2}-\kappa^{1}\right)-\left(\kappa^{1}\right)-\mu_{3}$ and $\left(\kappa^{2}\right)-\left(\kappa^{2}\right)-\mu_{2}$ (Chart $1(\mathrm{c})$ and (d)), linking three or two metal ions. In the pores, the guest molecules are protonated $4,4^{\prime}$-Hbipy

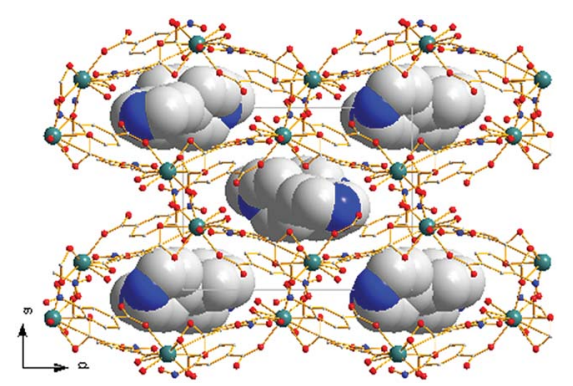

Fig. 6 3D porous structure of complex 2 , containing 4,4'-Hbipy guest molecules, in the ab plane. ligands, which exist via $\mathrm{H}$-bonds with the coordinated water molecules (Fig. 6).

\section{Crystal structure of 4}

In 4, the Tb1 ion is surrounded by six 2-ntp ${ }^{2-}$ ligands, with their carboxyl groups in chelate (O3, O15, O6A, and O8A) or monodentate $(\mathrm{O} 4, \mathrm{O} 10, \mathrm{O} 6$, and $\mathrm{O} 11)$ mode, and one coordinated water molecule (O16), which construct a one-capped square antiprism polyhedron (Fig. 7). As shown in Fig. 7b, O3, O15, O4, and 016 and O6, O11, O8A, and 010 form the square faces of the prism, and O6A is the capping atom. The $\mathrm{Tb}-\mathrm{O}$ bond lengths range from 2.338(5) to 2.724(6) $\AA$, and the $\mathrm{O}-\mathrm{Tb}-\mathrm{O}$ bond angles are in the range of $52.7(2)-149.15(18)$.

The dimer unit consists of two terbium ions with a $\mathrm{Tb} \cdots \mathrm{Tb}$ distance of $4.10 \AA$, which is less than in complexes 2 and 3 . These dimers are linked via 2-ntp ${ }^{2-}$ ligands in two coordination modes, $\left(\kappa^{2}-\kappa^{1}\right)-\left(\kappa^{2}\right)-\mu_{3}$ and $\left(\kappa^{1}-\kappa^{1}\right)-\left(\kappa^{1}\right)-\mu_{3}$ (Chart $1(e)$ and (f)), into a 3D framework, which differs from complexes 1-3 (Fig. 8a). As for guest molecules, long, soft, protonated 1,4- $\mathrm{H}_{2}$ bbi ligands are located in the cavities of the framework via $\mathrm{H}$-bonds with the coordination water molecules (Fig. 8b).

\section{Discussion relating to the structures}

The porous Tb-MOF series features different guest molecules $\left(\mathrm{H}_{2} \mathrm{O}, 4,4^{\prime}\right.$-bipy, 2,4-bipy and 1,4-bbi) (Chart 2), which are $\mathrm{N}$ -

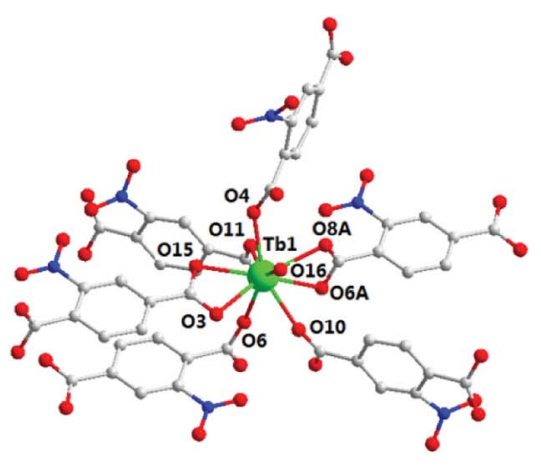

(a)

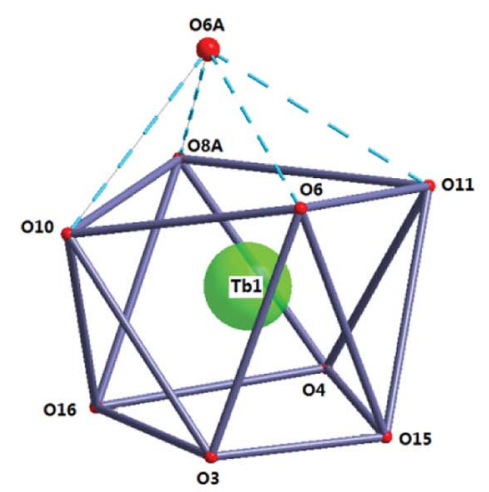

(b)

Fig. 7 The Tb1 coordination environment (a) and polyhedron (b) for complex 4. All $\mathrm{H}$ atoms are omitted for clarity. Symmetry code: (A) $1-$ $x, 1-y,-z$. 


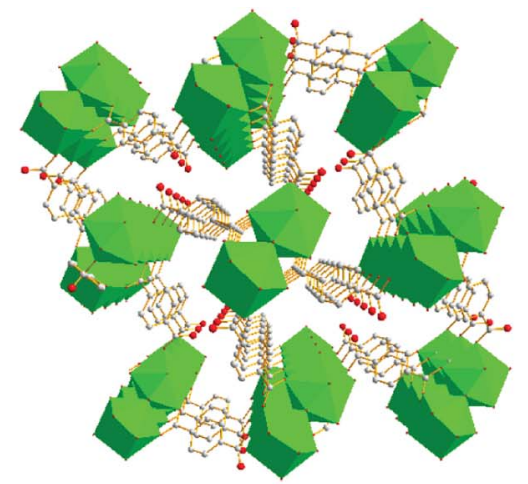

(a)

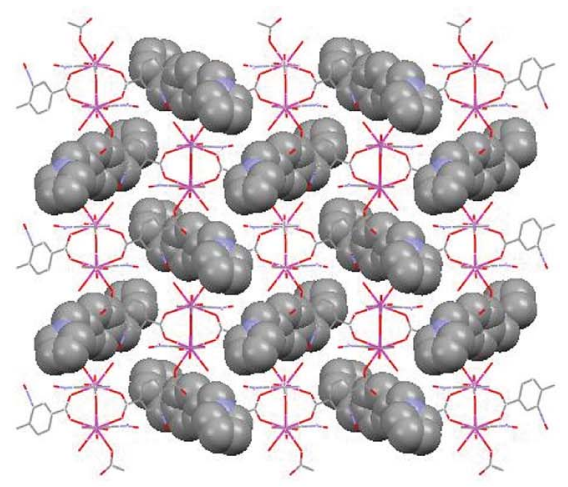

(b)

Fig. 8 (a) The porous structure along the $c$ axis, with nitro groups and $\mathrm{H}$ atoms omitted for clarity; and (b) the 3D structure, containing 1,4$\mathrm{H}_{2}$ bbi guests, of 4 .

donor long-chain ligands, except for $\mathrm{H}_{2} \mathrm{O}$ in 1. The frameworks of complexes 2-4 display negative charge and interacted with the protonated guest molecules, while 1 differs from these with an electrically neutral framework. From $\mathrm{H}_{2} \mathrm{O}$ to $4,4^{\prime}$-bipy to 1,4bbi, the guests in complexes 1-4 get bigger and longer, and the frameworks adjust their pores to contain these guests. We tried to use a longer guest than 1,4-bbi when preparing complexes

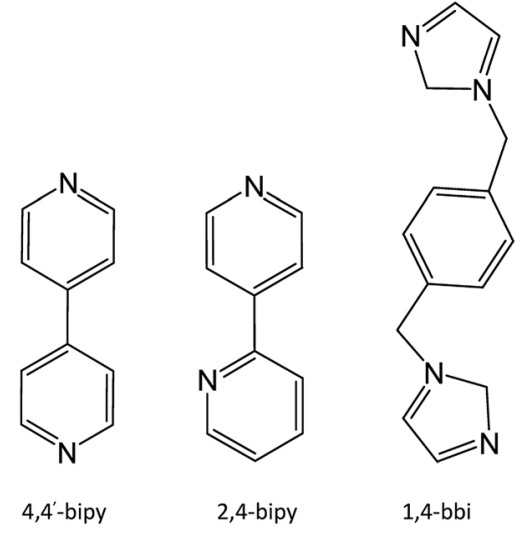

Chart 2 The $\mathrm{N}$-donor guest molecules used in complexes 2-4. but this failed, which might mean that pore sizes of these frameworks are limited. The existence of guest molecules might affect the coordination polyhedrons of metal ions and the coordination modes of the organic ligands. The host-guest system of Tb-MOFs has been reported in recent years. ${ }^{\mathbf{1 4}}$ The $\mathrm{Tb}$ (III) ion coordination number is eight for $\mathbf{1}$ and nine for $\mathbf{2 - 4}$, and the coordination polyhedrons change from a two-capped triangular prism to a three-capped triangular prism to a onecapped square antiprism, and are probably affected by the template molecules in the pores. The $2-n \mathrm{tp}^{2-}$ ligand adopts six coordination modes in these complexes: (a) $\left(\kappa^{2}-\kappa^{1}\right)-\left(\kappa^{1}-\kappa^{1}\right)-\mu_{4}$; (b) $\left(\kappa^{1}-\kappa^{1}\right)-\left(\kappa^{1}-\kappa^{1}\right)-\mu_{4} ;(\mathrm{c})\left(\kappa^{2}-\kappa^{1}\right)-\left(\kappa^{1}\right)-\mu_{3} ;(\mathrm{d})\left(\kappa^{2}\right)-\left(\kappa^{2}\right)-\mu_{2} ;(\mathrm{e})\left(\kappa^{2}-\kappa^{1}\right)-\left(\kappa^{2}\right)-$ $\mu_{3}$; and (f) $\left(\kappa^{1}-\kappa^{1}\right)-\left(\kappa^{1}\right)-\mu_{3}$. The 2 -ntp ${ }^{2-}$ ligand acts in a $\mu_{4}$ fashion to link four metal ions in 1 , but the $\mu_{2}$ and $\mu_{3}$ fashion are also probably affected by the N-donor guests in 2-4.

\section{Powder X-ray diffraction}

In order to confirm the phase purity of the bulk materials, powder X-ray diffraction (PXRD) patterns were recorded at room temperature for 1-4 (see Fig. S1 $\dagger$ ). Compared to simulated patterns from the single crystal data, although the experimental patterns have a few unindexed diffraction peaks and some peaks are slightly broadened, we can conclude that the bulk assynthesized crystalline materials represent complexes 1-4, due to their adequate similarity.

\section{Thermal stability behaviour}

In order to examine the thermal stabilities of complexes 1-4, we carried out thermogravimetric analyses (TGA) under an air atmosphere at a heating rate of $10{ }^{\circ} \mathrm{C} \mathrm{min}{ }^{-1}$, from 30 to $900{ }^{\circ} \mathrm{C}$ (Fig. S2 $\dagger$ ). The TGA curve for 1 exhibits that the first weight loss step of $11.2 \%$ began from $234{ }^{\circ} \mathrm{C}$, which indicates the high stability of the complex, and could be attributed to the loss of one coordinated and two lattice water molecules (calc. 10.8\%). The second step might be the complete collapse of the whole skeleton of $\mathbf{1}$, from 369 to $798^{\circ} \mathrm{C}$ (Fig. S2a $\dagger$ ). The TGA curves of complexes 2-4 are similar and show one-step decomposition (Fig. S2b-S2d †). The complex possesses high stability in relation to the initial decomposition from $242{ }^{\circ} \mathrm{C}$ for 2 . At $304{ }^{\circ} \mathrm{C}$ for 2 , there is point of inflexion, with a weight loss of $79.8 \%$, which could be assigned to the release of the guest molecule $4,4^{\prime}$-bipy (calc. 79.3\%). Following the release, the framework of complex 2 decomposed. Complex 3 begins to decompose from $270{ }^{\circ} \mathrm{C}$, and then the framework of 3 is destroyed up to $550{ }^{\circ} \mathrm{C}$ with a weight loss of $25.3 \%$ which can be considered as decomposition to yield terbium oxide (calc. $24.31 \%$ ). For complex 4 , thermal decomposition begins from $282{ }^{\circ} \mathrm{C}$, and the skeleton is broken down up to $840{ }^{\circ} \mathrm{C}$, with the residual weight $28.8 \%$ being attributed to $\mathrm{Tb}_{2} \mathrm{O}_{3}$ (calc. $25.68 \%$ ).

\section{Luminescence properties}

The fluorescence spectra of complexes 1-4 were obtained in the solid state at room temperature. From Fig. 9, we can see that all the emission spectra of these complexes exhibit the characteristic peaks of the terbium ion $\left(\lambda_{\mathrm{ex}}=487 \mathrm{~nm}\right)$ at $541 \mathrm{~nm}, 580 \mathrm{~nm}$, $617 \mathrm{~nm}$ and $670 \mathrm{~nm}$, corresponding to ${ }^{5} \mathrm{D}_{4} \rightarrow{ }^{7} \mathrm{~F}_{5},{ }^{5} \mathrm{D}_{4} \rightarrow{ }^{7} \mathrm{~F}_{4}$, 
${ }^{5} \mathrm{D}_{4} \rightarrow{ }^{7} \mathrm{~F}_{3}$ and ${ }^{5} \mathrm{D}_{4} \rightarrow{ }^{7} \mathrm{~F}_{2}$ transitions. The excitation spectra of the four complexes each show one single peak at $487 \mathrm{~nm}\left(\lambda_{\mathrm{em}}=\right.$ $541 \mathrm{~nm}$ ) (Fig. S3†), the intensities of which are similar to those of the emission peaks. The intensities of emissions of these complexes are different, especially complex 3 , which shows drastic quenching, which means that the existence of guest molecules might affect ligand-to-metal energy transfer (LMET) and result in fluorescence enhancing or quenching. The luminescence quenching mechanism induced by guests might be explained through a donor-acceptor electron transfer effect from ligands to metal centers. ${ }^{15}$ The effects of guest molecules on the fluorescence intensities of Tb-MOFs have been reported. ${ }^{16}$ We have analyzed carefully the structures and luminescence properties of these Tb-MOFs, and found that different interactions between the guest molecules and the organic ligand 2-ntp ${ }^{2-}$ might be the key to the effects on lanthanide emissions. It is well known that the luminescence properties of lanthanide complexes mainly depend on the coordination environments of the metal ions contributing to the energy transfer from $\mathrm{L}$ ligands to $\mathrm{Tb}$ (III) ions. For these complexes, the guest molecules exist in the pores and have no interactions with the $\operatorname{Tb}(\mathrm{III})$ ions directly, but they interact with the organic ligands and might affect the energy transition to $\mathrm{Tb}$ (III) ions from the 2-ntp ${ }^{2-}$ ligands. The intermolecular interactions between the guests and 2-ntp ${ }^{2-}$ ligands are $\mathrm{H}$-bonding and $\pi-\pi$ packing interactions, but the $\mathrm{H}$-bonds are similar to the close distances of $\mathrm{N} \cdots \mathrm{O}$ atoms, at about $2.60 \AA$, so we investigated the $\pi-\pi$ packing interactions. From the structural factors, two pyridine rings of the guest 2,4-bipy in complex 3 have good coplanarity with the smallest dihedral angle of $19.15^{\circ}$ (other guests have bad coplanarity, as shown in Fig. $\mathrm{S} 4 \dagger$ ), which results in stronger $\pi-\pi$ packing interactions with 2-ntp ${ }^{2-}$ ligands than the other guests in $\mathbf{2}$ and $\mathbf{4}$ have. The weak bonding between 2,4bipy and $2-$ ntp $^{2-}$ might partly quench the singlet and triplet excited stated of the ligand-to-Tb optically active core, and might decrease the efficiency of energy transfer, causing the decreasing luminescence intensity of 3.

The fluorescence lifetimes of these complexes have been determined, in which the processes of decay all involve two



Fig. 9 The emission spectra of complexes 1-4. components. The lifetime of complex 1 is $\tau=323.73 \mu$ s with a quantum yield of $65.59 \%, \tau=334.41 \mu$ s with a quantum yield of $59.56 \%$ for $2, \tau=443.42 \mu$ s with a quantum yield of $26.60 \%$ for 3 , and $\tau=374.67 \mu$ s with a quantum yield of $49.43 \%$ for 4 . From the lifetimes and quantum yields of these complexes, we could find that the lifetimes of complexes 2-4 show varying degrees of improvement caused by the existence of guest molecules, but the quantum yields decrease compared with complex 1.

\section{Conclusions}

In summary, a series of porous Tb-MOFs with 2-nitroterephthalate, featuring different template guests $\left(\mathrm{H}_{2} \mathrm{O}(\mathbf{1}), 4,4^{\prime}\right.$ Hbipy (2), 2,4-Hbipy (3), and 1,4- $\mathrm{H}_{2}$ bbi (4)), has been reported. The 2-ntp ${ }^{2-}$ ligand adopts six coordination modes in these complexes and they extend into 3D porous frameworks containing different guest molecules. The terbium(III) ions lie in eight/nine-coordination environments with two-capped triangular prism (1), three-capped triangular prism (2 and 3 ), and one-capped square antiprism (4) polyhedron configurations, which may be affected by the different guest molecules. The luminescence emission spectra exhibit the characteristic peaks of the terbium ion in the solid state, and the intensities have been influenced by the existence of guest molecules.

\section{Conflicts of interest}

There are no conflicts to declare.

\section{Acknowledgements}

This work was supported by the National Natural Science Foundation of China (No. 21573189), and the Natural Scientific Research Foundation of National Undergraduate Training Programs of Innovation and Entrepreneurship (No. 201510719256 and D2016019).

\section{Notes and references}

1 Y. P. Wu, W. Zhou, J. Zhao, W. W. Dong, Y. Q. Lan, D. S. Li, C. H. Sun and X. H. Bu, Angew. Chem., Int. Ed., 2017, 56, 13001; S. Horike, R. Matsuda, D. Tanaka, S. Matsubara, M. Mizuno, K. Endo and S. Kitagawa, J. Am. Chem. Soc., 2006, 128, 4222; L. Wang, Y. Z. Han, X. Feng, J. W. Zhou, P. F. Qi and B. Wang, Coord. Chem. Rev., 2016, 307, 361381; M. Yoon, R. Srirambalaji and K. Kim, Chem. Rev., 2012, 112, 1196.

2 Y. Li, S. S. Zhang and D. T. Song, Angew. Chem., Int. Ed., 2013, 52, 710; A. J. Timmons and M. D. Symes, Chem. Soc. Rev., 2015, 44, 6708; C. B. He, D. M. Liu and W. B. Lin, Chem. Rev., 2015, 115, 11079.

3 M. C. Bernini, N. Snejko, E. G. Puebla, E. V. Brusau, G. E. Narda and M. A. Monge, Inorg. Chem., 2011, 50, 5958; Y. F. Yue, Z. A. Qiao, P. F. Fulvio, A. J. Binder, C. C. Tian, J. H. Chen, K. M. Nelson, X. Zhu and S. Dai, J. Am. Chem. Soc., 2013, 135(26), 9572. 
4 V. Chandrasekhar, P. Bag and E. Colacio, Inorg. Chem., 2013, 52, 4562; X. L. Mei, X. F. Wang, J. J. Wang, Y. Ma, L. C. Li and D. Z. Liao, New J. Chem., 2013, 37, 3620; B. Zhang, X. D. Zheng, H. Su, Y. Zhu, C. X. Du and M. P. Song, Dalton Trans., 2013, 8571.

5 D. Kovacs, X. Lu, L. S. Meszaros, M. Ott, J. Andres and K. E. Borbas, J. Am. Chem. Soc., 2017, 139, 5756; S. P. Chen, Y. X. Ren, W. T. Wang and S. L. Gao, Dalton Trans., 2010, 1552-1557.

6 G. W. Xu, Y. P. Wu, W. W. Dong, J. Zhao, X. Q. Wu, D. S. Li and Q. C. Zhang, Small, 2017, 13, 1602996; G. W. Xu, Y. P. Wu, W. W. Dong, J. Zhao, D. S. Li and J. Zhang, Inorg. Chem., 2016, 55, 10114.

7 S. F. Du, C. Q. Ji and X. L. Xin, J. Mol. Struct., 2017, 1130, 565; J. M. Zhou, W. Shi, N. Xu and P. Cheng, Cryst. Growth Des., 2013, 13, 1218; Y. X. Ren, H. Y. Ma, F. Fu, Z. L. Zhang and Y. R. Zhang, J. Inorg. Organomet. Polym., 2013, 23, 646.

8 A. E. Platero-Prats, N. Snejko, M. Iglesias, A. Monge and E. Guti errez-Puebla, Chem.-Eur. J., 2013, 19, 15572; Y. X. Ren, S. S. Xiao, L. C. Li and X. J. Zheng, Inorg. Chem. Commun., 2012, 16, 8; J. Zhang, T. Wu, S. Chen, P. Feng and X. Bu, Angew. Chem., Int. Ed., 2009, 51, 3486; Z. Chen, B. Zhao, P. Cheng, X. Q. Zhao, W. Shi and Y. Song, Inorg. Chem., 2009, 48, 3493.
9 H. Seko, K. Tsuge, A. Igashira-Kamiyama, T. Kawamoto and T. Konno, Chem. Commun., 2010, 46, 1962; M. H. Mir and J. J. Vittal, Angew. Chem., Int. Ed., 2007, 46, 5925; J. R. Li, D. J. Timmons and H. C. Zhou, J. Am. Chem. Soc., 2009, 131, 6368.

10 G. M. Sheldrick, SADABS, A Program for Empirical Absorption Correction of Area detector Data, University of Göttingen, Germany, 1997.

11 G. M. Sheldrick, SHELXS-97, Program for Crystal Structure Solution, University of Göttingen, Germany, 1997.

12 G. M. Sheldrick, SHELXL-97, Program for Crystal Structure Refinement, University of Göttingen, Germany, 1997.

13 G. X. Wen, M. L. Han, X. Q. Wu, Y. P. Wu, W. W. Dong, J. Zhao, D. S. Li and L. F. Ma, Dalton Trans., 2016, 15492.

14 I. A. Ibarra, T. W. Hesterberg, J. S. Chang, J. W. Yoon, B. J. Holliday and S. M. Humphrey, Chem. Commun., 2013, 49, 7156; J. M. Zhou, W. Shi, H. M. Li, H. Li and P. Cheng, J. Phys. Chem. C, 2014, 118, 416.

15 J. X. Ma, X. F. Huang, X. Q. Song and W. S. Liu, Chem.-Eur. J., 2013, 19, 3590; J. N. Hao and B. Yan, J. Mater. Chem. A, 2014, 2, 18018.

16 J. Zhao, Y. N. Wang, W. W. Dong, Y. P. Wu, D. S. Li and Q. C. Zhang, Inorg. Chem., 2016, 55, 3265. 